\title{
183. Sur une nouvelle espèce d'Hemiclepsis, parasite d'un crabe fluviatile de Chine.
}

\author{
Par Asajiro OKA, M.I.A. \\ Institut zoologique, Ecole normal supérieure, Tokyo.
}

(Comm. Dec. 2, 1928.)

Grâce à l'amabilité de M. Tung Yüh-Mou, qui les a recueillis, j'ai pu examiner récemment plusieurs exemplaires d'une Glossiphonide, qui vit en parasite sur un Crabe fluviatile (Potamon sp.) de Chine. L'animal appartient au genre Hemiclepsis et se distingue des autres espèces très aisément par le nombre et la disposition de ses yeux. En outre, c'est la première fois, à ma connaissance, qu'on ait observé une Hirudinée qui se rencontre régulièrement, sinon exclusivement, dans la cavité branchiale des Crustacés d'eau douce.

\section{Hemiclepsis cancricola nov. sp.}

Cette espèce est certainement des plus petites, les plus grands exemplaires ne mesurant que $9 \mathrm{~mm}$ de longuear et $2 \mathrm{~mm} 5$ de largeur. La forme du corps est à peu près identique à celui de l'Hemiclepsis smaragdina, décrite par moi dans le dernier numéro de ce journal..1) Ainsi que chez cette dernière, les ventouses antérieure et postérieure sont relativement larges, et, quand l'animal est étendu, elles sont toutes deux plus larges que le corps. Quelques-uns portaient une quinzaine environ de petits sous le ventre; chez eux, la face ventrale était naturellement concave.

Les papilles dorsales sont, comme chez l'Hemiclepsis smaragdina, fort peu développées, de sorte que la surface du corps est presque lisse dans certains individus. De même, la coloration du corps est très faible, les plus grands exemplaires seuls montrant des cellules pigmantaires brunes, peu nombreuses et espacées, sur toute la longueur $\mathrm{du}$ dos, tandis que les petits en sont tout à fait dépourvus.

Quant à l'annulation du corps, on trouve une coincidence à peu près complète entre cette espèce et l'Hemiclepsis smaragdina, la seule différence portant sur le somite XXV, qui est ici uniannelé. Il y a donc en tout 68 anneaux en avant de la ventouse postérieure.

La région céphalique est constituée par les somites $\mathrm{I}$ à $\mathrm{V}$, dont le dernier correspond à la lèvre postérieure de la ventouse. L'orifice buccal est percé dans la partie antérieure de cette dernière. 4 (1928).

1) Oka, A. Description de deux espèces japonaises de Glossiphonia. Proc. 


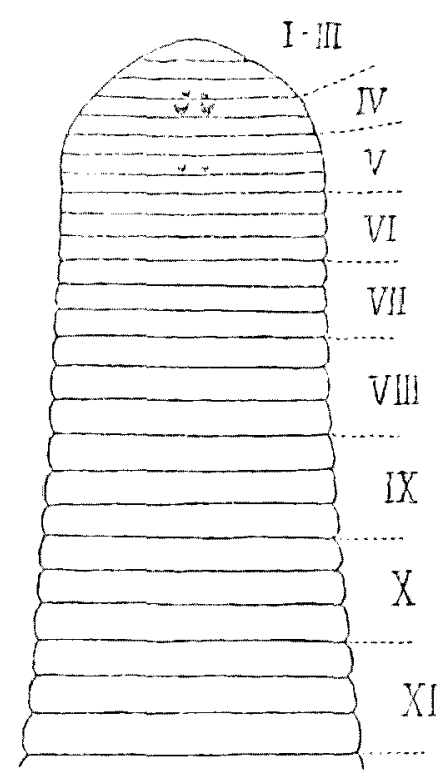

A

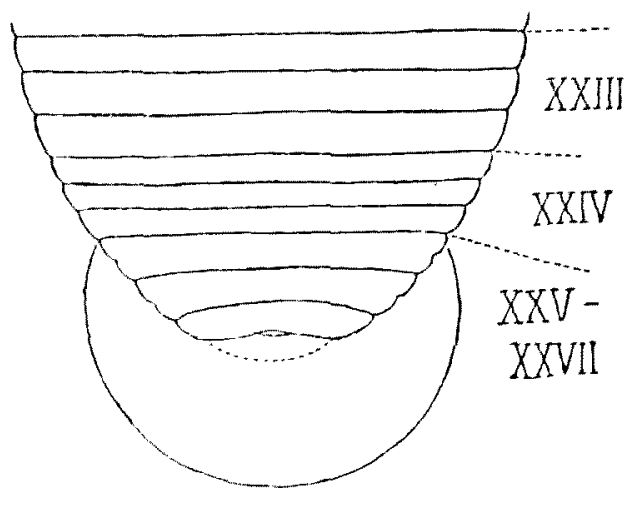

B

\section{Hemiclepsis cancricola}

A. Somites I-XI, vus par la face dorsale.

B. Somites XXIII-XXVII, vus par la face dorsale.

Les yeux sont au nombre de trois paires, portés par les anneaux 3, 4 et 7 , c'est-à-dire par le somite III, uniannelé, par l'anneau antérieur du somite IV, biannelé, et par le deuxième anneau du somite $V$, triannelé. Ils sont de taille inégale, ceux de la seconde paire étant de beaucoup plus grands que les autres. Tous sont dirigés en avant.

Les orifices génitaux occupent la situation habituelle: l'orifice mâle s'ouvre dans l'interstice des somites XI et XII, l'orifice femelle entre les deux premiers anneaux du somite XII. Le clitellum n'etait pas apparent.

L'anus se trouve en arrière du dernier anneau.

La ventouse postérieure est circulaire, discoïde et quasi-pédonculée.

En ce qui concerne l'anatomie intérieure, je me borne à mentionner qu'il y a six paires de testicules, alternant régulièrement avec les coecums gastriques. Comme chez toutes les autres espèces du même genre, ces derniers sont au nombre de sept paires et symétriquement ramifiés, ceux de la derniere paire s'étendant jusqu' au somite XXIV.

Tous mes exemplaires proviennent de Pan-chi, Feng-hua, prov. Che-chiang. Un lot, comprenant 11 individus, a été recueilli en août 1924 ; un autre, comptant plus d'une trentaine d'individus, grands et petits, en août de cette année (1928). Il semble que notre Hirudinée ne soit pas rare dans cette localité, car M. Tung m'a dit qu'il en a trouvé sur plus de $10 \%$ des crabes qu'il a examinés, quelquefois jusqu'à 41 exemplaires sur un seul crabe. 